\title{
A Bayesian Structural Time Series Model for Assessing Road Traffic Accidents during COVID-19 Period
}

\author{
Armana Huq ${ }^{1}$, Shahrin Islam² \\ ${ }^{1}$ Assistant Professor, Bangladesh University of Engineering and Technology (BUET), Dhaka 1000, Bangladesh \\ ahuq002@fiu.edu; ashuq@ari.buet.ac.bd \\ ${ }^{2}$ Lecturer, Daffodil International University (DIU), Dhaka 1207, Bangladesh \\ Shahrin.ce@diu.edu.bd
}

\begin{abstract}
This is a systematic study to understand the effect of the implementation and removal of restrictive measures on road accidents statistics, using newspaper-based database. The time frame of data is between 1st January 2020 to 31 st December 2020. In this period, 26th of March was the first day of official lockdown and 30th of May was the day of lockdown withdrawal in Bangladesh. However, the first COVID-19 affected case was identified on 8th of March followed by the closure of educational institutions on 18th of March of the same year. In the selected time frame, the total number of accidents was recorded 3,069 with total injuries and fatalities being 593 and 3,570 respectively. The new confirmed cases, death and recovered cases of Coronavirus affected people were collected from Humanitarian Data Exchange, 2020 to understand their correlations with accident statistics. This study considered two interventions: starting of lockdown and reopening. Results suggest that the number of daily traffic accidents and related fatalities increased by about the same amount after the reopening as they had decreased due to the lockdown. This study also conducted the two-sample t-tests to find variations of mean in overall incident cases, casualties, and injuries on a daily basis before, during, and after the mandatory lockdown. It is found that there is a significant reduction in total road traffic accidents in the lockdown period than pre-lockdown period, followed by a slight increase in the post lockdown period. In case of fatalities, before and during the lockdown, a drastic reduction has been observed from 13.94 to 5.80 with p-value <2.2e-16, followed by an increase. Finally, statistics of mean injuries have also experienced a steep drop during the lowdown period and rise after the lockdown. The effect size of the obtained means is also evaluated using Cohen's $d$ test.
\end{abstract}

Keywords: Road Accident, Time Series Model, Lockdown, COVID-19, Bangladesh.

\section{Introduction}

As of April 2021, COVID-19 has affected more than hundred and twenty-eight million people in 219 countries around the world, killing approximately two million eighty thousand people. Along with our health, it has impacted nearly every facet of life. These impacts have primarily been brought by the imposed lockdown which has been recognized as one of the most effective techniques for preventing coronavirus transmission by imposing restrictions on intercity and country wide transportation [1]. Mobility has been no exception in this regard and a sharp decline is observed in the lockdown areas. Though restricted mobility has found to be having a negative impact on the economy of a country [2], [3], it has a positive impact on the number of road accidents involving injuries and deaths.

Apart from the primary causes of accidents such as lack of law enforcement, drugs and alcohol, inexperience, distractions etc., a significant drop has been observed in accidents and related casualties in many parts of the world with the restriction in mobility [4], [5], [6], [7]. Unfortunately, accident rates have risen in many countries around the world since the lockdown was lifted [8], [9], [10]. Despite the fact that traffic accidents are caused by a variety of causes, the decrease in traffic accidents is notable due to lower traffic volumes [11].

The first COVID-19-affected case was discovered in Bangladesh on March 8th, 2020 [12]. The Government of Bangladesh has announced the closure of educational institutions on March 18th, followed by a countrywide lockdown from March 27th to May 30th in the year of 2020 [13], [14]. However, the lockdown was not applied to trucks, covered vans, or vehicles transporting drugs, fuel, or perishable goods [14]. During the year, a total of 3,069 accident cases were registered across Bangladesh, with 593 injuries and 3,570 fatalities, respectively. The total number of accidents associated with injuries was 104 and no injuries only 12. Unfortunately, the number of accidents causing both fatalities and injuries was 2,953 (96.13\% of the total accidents), among which 1,105 accidents were reported to be fatal without any casualties [15]. According to the police, at least 4,138 people died and 4,411 were injured in 4,147 incidents in 2019, compared to 2,635 deaths and 1,920 injuries in 2,609 accidents in 2018 [16]. The figures for 2018 and 2019 are significantly higher than those for 2020, 
suggesting that the statistics for 2020 might have been higher than the previous two years if the lockdown had not occurred. Another study shows that after COVID-19 public transportation were badly affected. People without emergency did not go out of house, which eventually had a positive impact on roadway accidents [42].

Based on this context, our primary goal was to determine the effect of the COVID-19 lockout and reopening on traffic accidents focusing on Bangladesh. The reduction in the number of traffic incidents was investigated, as well as more detailed data, such as: (a) variations in mean value of total daily accident cases, casualties, and injuries aiming the period of before, during, and after the mandatory lockdown, (b) correlation of daily number of accidents, deaths, and injuries with the number of new COVID-19 affected cases, death cases, and recoveries, and (c) Causal Impact analysis including the counterfactual details. It is expected that this study would add to the increasing range of evidence about the effects of the COVID-19 pandemic on road safety.

\section{Literature Review}

During the COVID-19 outbreak, different governments have imposed different levels of restrictions in order to deter and monitor the virus's spread. These restrictions have a major effect on people's lives, social relationships, and economic circumstances. Fortunately, these regulations had a positive effect in a number of ways, including a reduction in air and water emissions as a result of decreased transport volume and industrial operations, a decline in road traffic injuries and violence, more time for people to relax with their family, enjoy sports, develop new skills, and a greater understanding of the strategies to be practiced to stop disease transmission and maintain good health [17].

Travel behaviors, frequency and mode choice can be influenced by fear of infection and perceived danger. During the pandemic, human transport associated with outdoor activities, in particular, has been severely impacted. The transition has been noticed from work and school to essential shopping, and mode preference has changed from public transportation and paratransit to private vehicles and active transportation [18], [19]. The number of daily and weekly trips also decreased significantly throughout the pandemic specially during the lockdown and the condition remained unchanged over the weekends as well [20]. Hence, the local traffic volumes dropped sharply, with the largest reductions occurring among children and students [21].

According to a number of studies, there is a link between reduced mobility and fewer accidents. According to a report conducted in the Italian province of Tarragona, total mobility decreased by 62.9 percent during COVID-19 lockout, while traffic accidents decreased by 74.3 percent, and the occurrence of major traffic accidents showed a significant decreasing pattern over the weekends [6]. Due to the strict law, improved residential mobility, and limitations on occupational and leisure activities during the lockout, a decrease in crash-fatality ratio has been observed by studying fifteen countries [22]. A report in Louisiana under lockdown stated that, the stay-at-home order resulted in a 47 percent reduction in total traffic incidents while accidents involving injury, distracted drivers, and ambulances, in particular, have decreased by 46 percent, 43 percent, and 41 percent, respectively [23]. Another study on Nepal confirmed the reduction in the number of road accidents, fatalities and injuries during the containment period. However, motorcycles were found to be responsible for the majority of the road deaths, accounting for 22.1 percent of all vehicles involved, accompanied by jeeps, tractors, trucks, and ambulances. According to additional observations, due to speeding, about 52.2 percent of the overall vehicles involved in the collisions were found to be out of the driver's control leaving the pedestrians at a vulnerable condition. [24]. The at-home order was released in Turkey in the middle of March, resulting in a 15\% decrease in on spot in only April. Accidents have decreased by 35 percent in terms of death or disability, death by 72 percent, and deaths by 19 percent, according to further research [25]. Apart from the accident statistics, the impact of a pandemic can also alter hotspots and the temporal distribution of accident frequency throughout the day resulting from changes in mobility patterns [26].

Several methods of analysis have been used to study the pattern of injuries and deaths during the mandatory shutdown. Based on the focus of this study, we would like to review a number of papers that analyzed accident statistics based on time series techniques. Qureshi et al. (2020), used Interrupted Time Series (ITS) and revealed that societal lockout policies impacted road traffic accidents that result in minor or no injuries, but not those that result in severe or fatal injuries [27]. Another ITS study by J.C. et al. (2021) found that the most significant change in the post-lockdown timeframe was in road crash mortality, with a decrease of 12.22 deaths per million men and 3.55 deaths per million women for every month [28]. 
From an ARIMA (Autoregressive Integrated Moving Average) time series analysis on speed-related fatalities undertaken by Inada et al. (2021), the reported ratio of speed-related fatalities to non-speed-related fatalities in case of motor vehicle accident was greater than the forecasted value in April 2020, suggesting that drivers who kept driving in the middle of the lockdown were more likely to violate traffic safety rules [29]. We were unable to locate any additional research on this topic to review. As a consequence, there is a void in the literature due to the absence of research on accident rates using time series methods, which could have assisted in assessing the trend, seasonality, and pattern variance of road accidents during the pandemic.

\section{Methods}

We performed an extensive analysis of the road accident data of Bangladesh collected from the Accident Research Institute of Bangladesh [15]. Bangladesh is an overly populated country with 1.26 thousand people per square kilometer [3]. According to the statistics of 2019, there are approximately 504,130.000 registered motor vehicles as authorized in the whole country [30]. The latest confirmed cases, death cases, and recovered cases of Coronavirus-affected people obtained from the Humanitarian Data Exchange, 2020, were used to figure out how they relate to accident statistics [31].

The study period is taken as 1st January 2020 to 31st December 2020. In this time frame, lockdown was imposed on 26th March to 30th May of the same year. This study aims at understanding the effect of lockdown and withdrawal of lockdown on the accident statistics separately. Hence, the selected time frame is divided into two main sections i.e., first intervention and second intervention.

\subsection{Bayesian Time Series Analysis}

Bayesian time series models are well-known for their ability to handle uncertainty with small sample sizes, which helps with future planning and decision-making. However, in some case, other conventional models i.e. ARIMA can outperform BSTS [32].

The "CausalImpact" package is used in RStudio software to carry out the numerical analysis [33]. This package is introduced by Google in 2015 that deals with the understanding of causal influence of a particular action within a time frame and assumes that finding a single causal effect is more powerful than a number of correlations. The statistical properties of the approach on synthetic data are demonstrated using a Markov chain Monte Carlo algorithm for model inversion. Causal impact can be referred to as anything that interacts with the natural environment [34].

The accident statistics is broken into three main categories such as total accidents, total fatalities and total injuries on a daily basis. While analysing the causal impact, the lockdown is considered as the first intervention with pre period between 1st January to 25th of March (the day before lockdown) and post period between 26th March (first day of lockdown) to 30th May (last day of lockdown). After that, the impact of lockdown withdrawal is considered as the second intervention with pre period between 26th of March (the first day of lockdown) to 30th May (last day of lockdown) and post period between 31th May (first day of reopening) to 31 st December.

\subsection{Statistical Analysis}

The correlation tests are conducted between six variables of this study i.e. daily total accidents, total fatalities, total injuries, new cases, death cases and recovered cases of COVID-19 affected people. For this test, "GGally" package is used in RStudio. The significance level is determined by Peason's significance level. We also used two-sample t-tests to look for differences in the mean of overall incident cases, casualties, and injuries on a daily basis before, during, and after the mandatory lockdown. For this test, the time period is divided into pre lockdown, during lockdown and after lockdown period. Cohen's d test is used to evaluate the effect size of the obtained mean [35]. The effect size can be classified as follows based on the Cohen's d concept: small $=0.2$ to less than 0.5 , medium $=0.5$ to less than 0.8 , large $=$ equal or greater than 0.8 .

\section{Results and Analysis}

Total road traffic accidents decreased significantly during the lockdown period compared to the pre-lockdown period (mean 12.01 versus 5.02, p-value 2.2e-16, effect size 1.80), followed by a marginal rise in the post-lockdown period (mean 
5.02 versus 7.99, p-value 1.256e-09, effect size 0.77). Again, in the case of fatalities, a significant drop from 13.94 to 5.80 with p-value $2.2 \mathrm{e}-16$ and effect size 1.55 was observed before and during the lockout, followed by a rise (5.80 versus 9.31 with $\mathrm{p}$-value $=1.002 \mathrm{e}-07$, effect size 0.65 ) after lifting the lockdown.

Finally, mean injury figures showed a sharp decrease during the lowdown era (mean 22.86 versus 4.20 , $p$-value $=5.631 \mathrm{e}-$ 1 , effect size 1.22) and a increase after the lockdown (mean 4.2 versus 10.33 , p-value $=6.506 \mathrm{e}-07$, effect size 0.52 ). In the correlation analysis, significant correlations are only evident among the accident statistics (significance level 0.001) and among the COVID-9 case statistics (significance level 0.001) separately. All the correlations between accidents and virus affected data did not surpass $10 \%$. From this result, we choose not to use new cases, death cases and recovered cases of COVID-19 as the covariates in the causal impact analysis of accident, fatalities and death statistics.

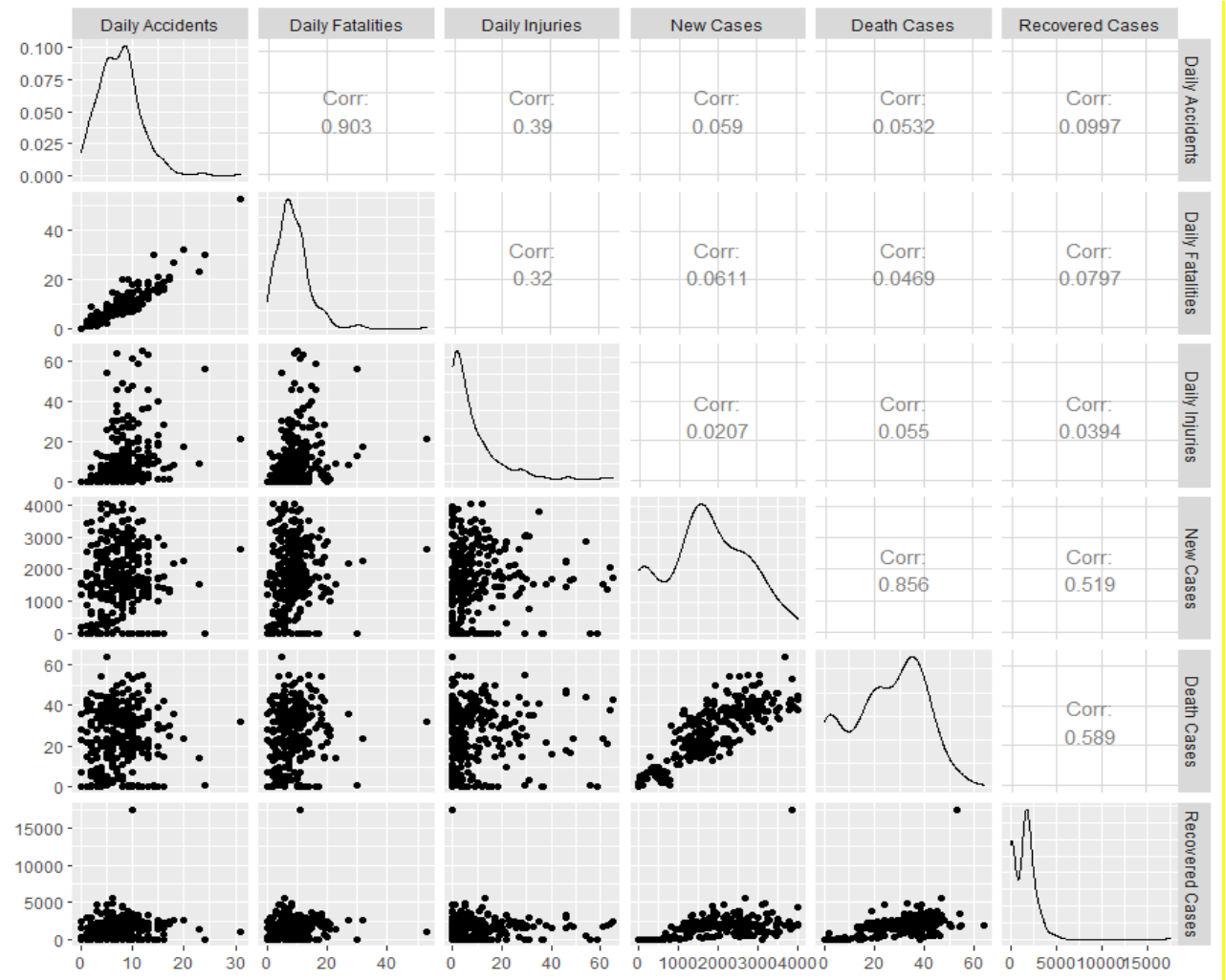

\subsection{Lockdown Impact Analysis}

Fig. 1: Correlation Plot.

The overall number of daily traffic accidents per day ranged from 0 to 25 during the first intervention period and 0 to 31 in the second intervention period. The fatalities ranged between 0 to 32 (first intervention) and 0 to 53 (second intervention) per day. Again, daily injuries varied between 0 to 92 and 0 to 65 during first and second intervention respectively. In Figure 1 and 2, the top graph named as "original" is showing the observed value in the zigzag pattern and the expected values in the dotted pattern.

The expected values which are also known as the counterfactual values, actually represents the condition without the impact. The second graph named as "pointwise" shows the difference between the observed and expected values which is helpful to understand the impact effect. The last graph which is the "cumulative" graph represents the total impacted value up to the last point of the dataset [36]. 


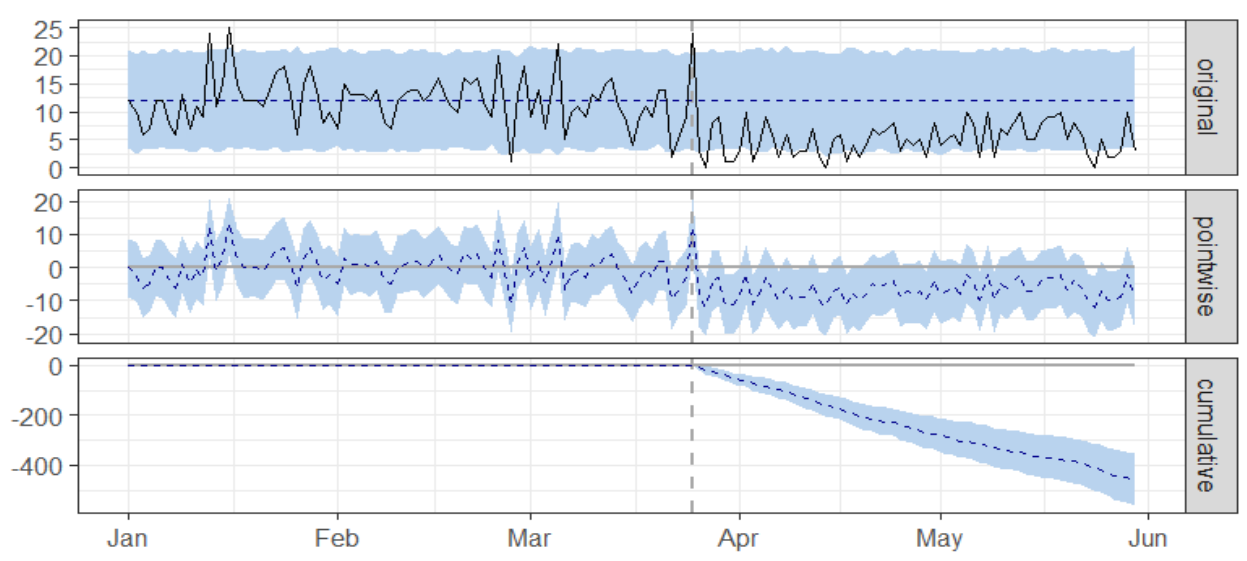

(a)
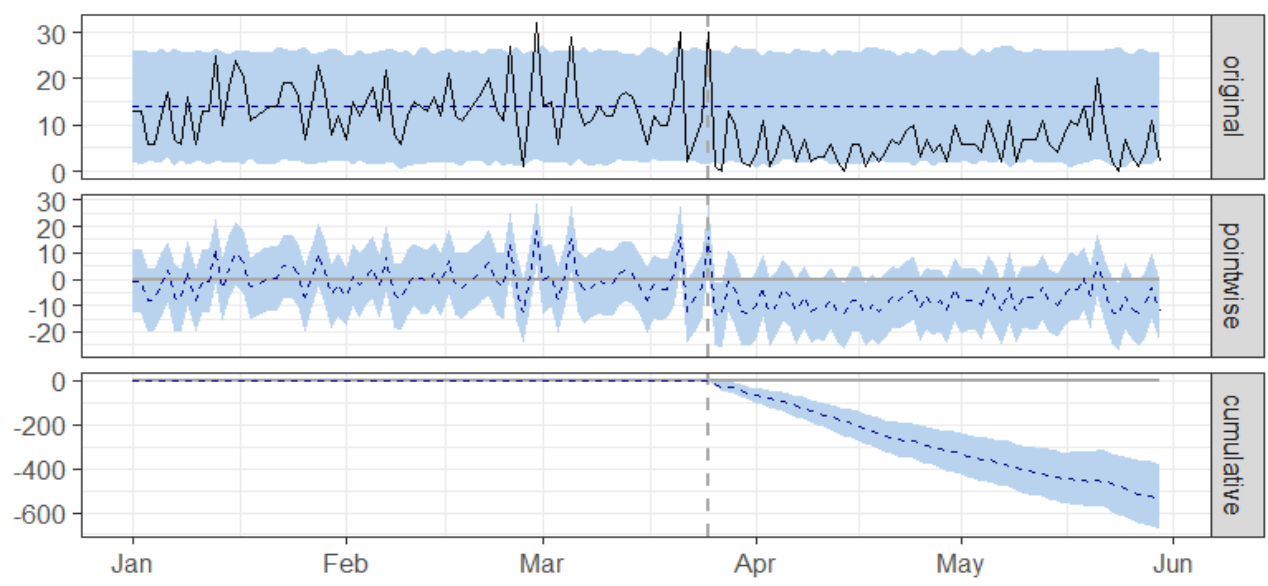

(b)

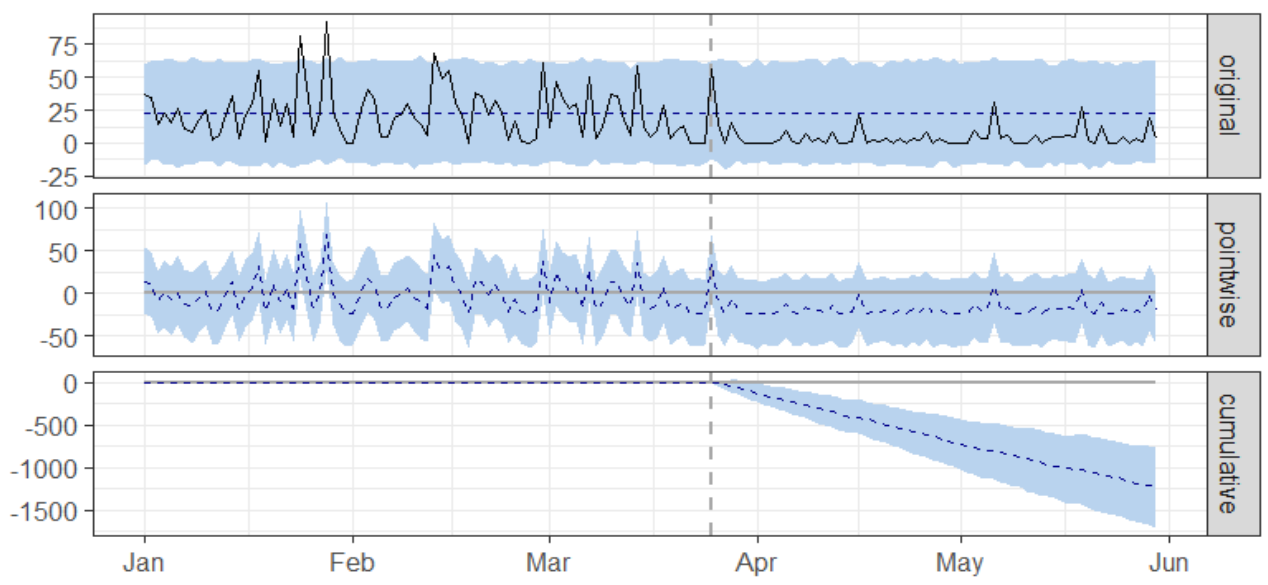

(c)

Fig. 2: Causal Impact Analysis of a) Total Daily Accident, b) Total Daily Fatalities and c) Total Daily Injuries during the first intervention period (Effects of Imposing Lockdown). 
During the post-intervention period, which is the lockdown period in this case, the daily accident is approximately 5.02 whereas in the absence of an intervention, we would have expected an average response of 11.98 (with 95\% confidence interval 10.35 and 13.51). The causal effect of lockdown is -6.96 with a $95 \%$ interval of $-8.50,-5.34$. Adding all data points during the lockdown period, the total daily accidents had an overall estimate of 331.00 whereas had the intervention not taken place, we would have expected a sum of 790.49 which yields a decrease of $-58 \%$ (95\% interval is $-71 \%,-45 \%$ ).

In case of daily fatalities, the estimate of with and without lockdown is 5.80 and 13.98 respectively $95 \%$ interval of this counterfactual prediction is 11.57 and 16.02. The causal effect is -8.18 with a $95 \%$ interval of $-10.22,-5.77$. During the lockdown, the sum of fatalities is 383 and the expected sum is 922.64 without the lockdown effect which results in $-58 \%$ ( $95 \%$ interval of this percentage is $-73 \%,-41 \%$ ) reductions in total fatalities.

Finally, while evaluating the injury data, we found that the approximate amount is 4.2 under the lockdown effect which is much lower than that of $22.84(95 \%$ interval $15.86,30.04)$ in the normal environment and the causal effect is -18.64 . The cumulative values are 227 and 1,507.42 with and without the lockdown effect which shows large differences than the accident and fatality estimates. Injury estimates decreased by $-82 \%$ in relative terms $(95 \%$ interval of this percentage is $-113 \%,-51 \%)$.

The negative effects found during the intervention phase in all the cases of total accidents, fatalities and injuries are statistically significant considering Bayesian one-sided tail-area probability $\mathrm{p}=0.001$.

\subsection{Reopening Impact Analysis}

The daily accident is approximately 7.99 during the reopened period while we would have predicted an average response of 5.04 in the absence of a closure (with 95 percent confidence interval 4.01 and 6.07). Reopening has a causal effect of 2.94, with a 95 percent confidence interval of 1.92 and 3.98. After combining all data points after the lockdown-free span, the total daily incidents were estimated to be 1,720 , while we would have predicted a total of 1080 if the intervention had not occurred, resulting in a +58 percent increase ( 95 percent interval +38 percent and +79 percent).

The figure 3 without and with lockout for regular casualties are 9.31 and 5.82, respectively, with a 95 percent interval of 4.52 and 7.26. With a $95 \%$ confidence interval of 2.05 to 4.79 , the causal effect is 3.49 . Without the reopening effect, the total number of fatalities is 2000, while the estimated total is 1,250 (the 95 percent interval for this forecast is 970 and 1,560) due to the lockdown withdrawal. The overall increase is $+60 \%$ with the $95 \%$ interval $+35 \%$ and $+82 \%$.

Finally, we discovered that the approximate number under the lockdown free effect is 10.33 , which is significantly higher than the value 4.45 (95 percent interval 2.29 and 6.73) throughout the controlled environment. The causal effect is 5.88, with a 95 percent interval of 3.60 and 8.04. After and before the reopening, the cumulative values are 2,200 and 960 (95 percent interval of this prediction is 490 and 1450) respectively with a relative increase of +132 percent (95 percent interval of this percentage is +81 percent and +181 percent).

Considering Bayesian one-sided tail-area likelihood $\mathrm{p}=0.001$, the positive results observed during the intervention process in all cases of total incidents, deaths, and injuries are statistically significant. They're also unlikely to be due to spontaneous fluctuations, according to the report. No residuals are found in these time series analyses. 


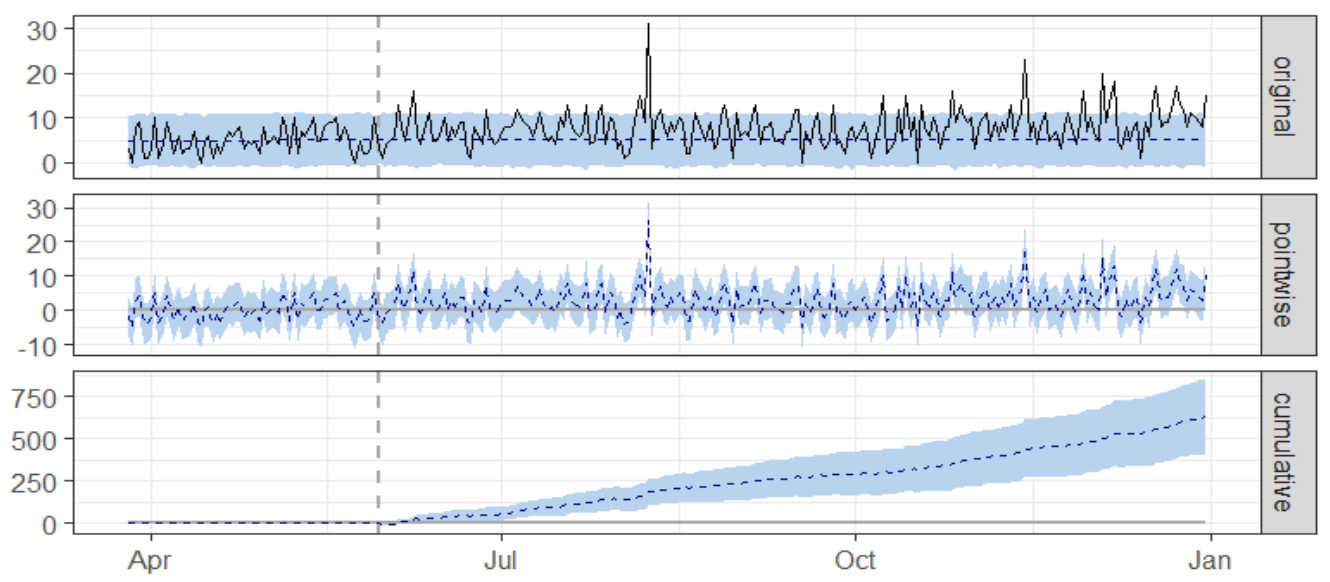

(a)

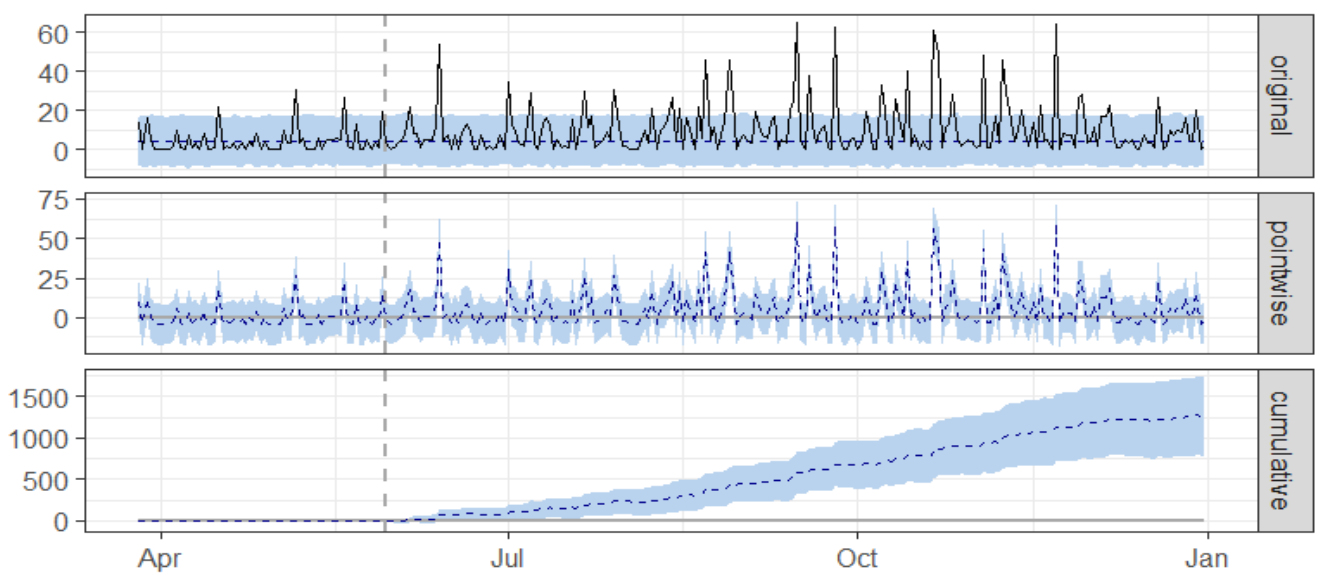

(b)

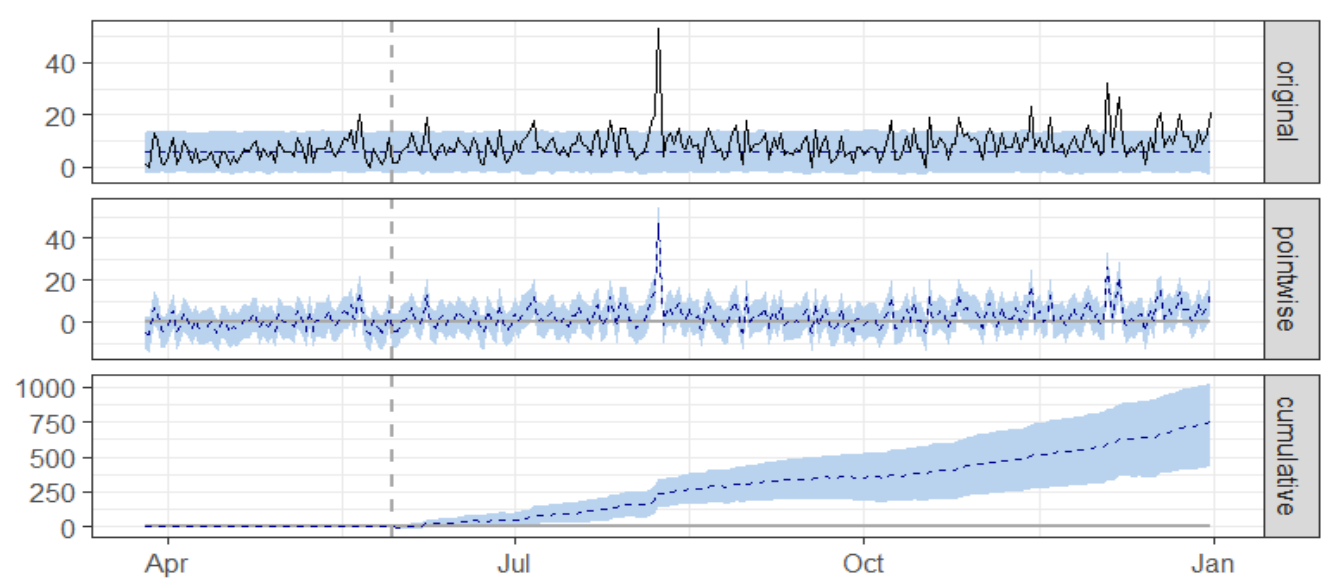

(c)

Fig. 3: Causal Impact Analysis of a) Total Daily Accident, b) Total Daily Fatalities and c) Total Daily Injuries during the second intervention period (Effects of Withdrawal of Lockdown) 


\section{Discussion}

We have observed that the total number of injuries and fatalities have declined alongside the total number of accidents during the lockdown period than the pre lockdown period. The exemptions provided for transporting necessary goods can be seen as the reason for the low number of accidents found during lockout. Although the figures increased after the mobility restrictions were lifted, they did not reach the levels observed prior to the lockdown, as evidenced by the mean analysis. Injury figures show the most variation, with the number of injuries after lockdown being less than half of what it was before lockdown. Although the actual reason behind the lesser non-fatal injuries after the lockdown is unclear, it may be attributed to the closing of educational institutions, which accounts for a significant portion of the total trip.

The coronavirus affected cases, death cases, and recovery cases have little effect on the regular statistics of total fatalities, mortalities, and injuries during the pandemic period, despite the fact that average mean values have shown variations as a result of the lockdown. There was no substantial link between incidents and virus-affected data in any of the correlations.

Peaks were observed shortly after the lockdown began, as people rushed back to their families in their hometowns [37]. This situation did not last long and the lockdown period has experienced a massive road incident declination which is evident from the counterfactual analysis [6]. However, the numbers did not reach zero because drivers have a tendency to speed up in the zones of lower traffic volumes and law enforcement and violate traffic legislation in order to save time and resources [24], [29]. Another research concluded that during the lockdown period, traffic volumes do not have a total effect on collisions and deaths, but that road configuration and driving habits do [38]. Apart from speeding up, drivers are also seen to use mobile phones during the peak of pandemic [39]. Also, the significant increase in traffic speed during the COVID-19 period is more noticeable on urban highways than on rural highways [7]. Hence, implementation of new speed as per the requirement should be useful to tackle this situation [39]. The daily traffic accident and fatalities statistics increased approximately by the same amount as they had decreased due to the lockdown. As a result, these incident rates can be anticipated to rebound to pre-pandemic peaks and patterns at some stage in the future. However, the injury statistics increased by a much higher amount than it had decreased.

\section{Conclusion}

Containment strategies for the COVID-19 pandemic have had huge ramifications in the transportation sector. The current research explores the influence of the COVID-19 pandemic on traffic accident figures. Both lockdown and lockdownfree phases have been considered in order to get a clear view. The trend of changes in road accidents before and after COVID19 is analyzed, as well as the impact of COVID-related cases. From the study, it is certain that reduced mobility due to lockdown has a positive impact on traffic accidents. However, this is not for a longer duration. Suspending the lockdown can restore things to the previous situation within a period of time. In Bangladesh, as an accident-prone country, the number of accidents can be reduced by implementing infrastructure that facilitates lower-density traffic conditions. Although less congestion may encourage drivers to break traffic laws, so an optimal situation is required to establish. The information gained from this study could be used by government officials to reschedule transportation operations with the intention of minimising accidents.

This study is only focused on the overall number of accidents occurred in the pre, during and post lockdown period. However, the lockdown is not strictly followed in many parts of the country especially in the small towns and villages which rely on unauthorized local vehicles [40]. Also, people tend to use a more non-motorized mode of transport especially in the less densely populated regions [41]. Hence, the contribution of unauthorized vehicles and non-motorized vehicles can be evaluated in the future studies. Also, the estimates of vehicle associations exempted from countrywide restrictions can also be considered. Due to COVID-19, we are experiencing a world that is never seen before. As a result, the findings of the study based on the pandemic situation may differ from those of previous research and generalization with the other contemporary studies is desired. 


\section{References}

[1] "COVID-19 spread and inter-county travel: Daily evidence from the U.S," Transportation Research Interdisciplinary Perspectives, vol. 8, p. 100244, Nov. 2020, Accessed: Mar. 31, 2021. [Online].

[2] "The COVID-19 dilemma: mobility and economy." https://www.caixabankresearch.com/en/economicsmarkets/activity-growth/covid-19-dilemma-mobility-and-economy (accessed Mar. 30, 2021).

[3] "Measuring the Economics of a Pandemic: How People Mobility depict Economics? An Evidence of People's Mobility Data towards Economic Activities." https://www.imf.org/-/media/Files/Conferences/2020/8th-stats-forum/paperrendra-putra-and-silvia-arini.ashx (accessed Mar. 30, 2021).

[4] "Novel Coronavirus (COVID-19) Cases Data." https://data.humdata.org/dataset/novel-coronavirus-2019-ncov-cases (accessed Mar. 24, 2021).

[5] "What are the factors that contribute to road accidents? An assessment of law enforcement views, ordinary drivers' opinions, and road accident records," Accid. Anal. Prev., vol. 115, pp. 11-24, Jun. 2018, Accessed: Mar. 31, 2021. [Online].

[6] "COVID-19 lockdown and reduction of traffic accidents in Tarragona province, Spain," Transportation Research Interdisciplinary Perspectives, vol. 8, p. 100218, Nov. 2020, Accessed: Mar. 30, 2021. [Online].

[7] F. Shilling and D. Waetjen, "Special report: Impact of COVID19 on California traffic accidents," Apr. 2020, Accessed: Mar. 30, 2021. [Online]. Available: https://trid.trb.org/view/1700419.

[8] D. Wilde, "Car accident rates climbing sharply after plummeting during lockdown," Motor1.com UK, Jul. $06,2020$. https://uk.motor1.com/news/432231/car-accident-rates-up-after-plummeting-lockdown/ (accessed Mar. 30, 2021).

[9] "Road accidents spike after govt eases lockdown in Tamil Nadu." https://timesofindia.indiatimes.com/city/trichy/roadaccidents-spike-after-govt-eases-lockdown-in-state/articleshow/76311306.cms (accessed Mar. 30, 2021).

[10] S. Biswas, "Road rage and accidents on the rise post lockdown," Sep. 09, 2020. https://timesofindia.indiatimes.com/lifestyle/spotlight/road-rage-and-accidents-on-the-rise-post-lockdown/articleshow/78009192.cms (accessed Mar. 30, 2021).

[11] "Relationship between crash rate and hourly traffic flow on interurban motorways," Accid. Anal. Prev., vol. 34, no. 5, pp. 619-629, Sep. 2002, Accessed: Mar. 30, 2021. [Online].

[12] R. Paul, "Bangladesh confirms its first three cases of coronavirus," Reuters, Mar. 08, 2020. https://www.reuters.com/article/us-health-coronavirus-bangladesh-idUSKBN20V0FS (accessed Mar. 31, 2021).

[13] "Bangladesh closes all educational institutions till March 31," Mar. $16,2020$. https://www.dhakatribune.com/bangladesh/dhaka/2020/03/16/govt-directs-shutting-all-educational-institutions-mach17-to-31 (accessed Mar. 31, 2021).

[14] "Bangladesh imposes total lockdown over COVID-19." https://www.aa.com.tr/en/asia-pacific/bangladesh-imposestotal-lockdown-over-covid-19/1778272 (accessed Mar. 31, 2021).

[15] “Accident Research Institute - Accident Research Institute.” https://ari.buet.ac.bd/ (accessed Mar. 25, 2021).

[16] "Numbers of road accidents, fatalities shoot up in Bangladesh." https://www.newagebd.net/article/99344/numbers-ofroad-accidents-fatalities-shoot-up-in-bangladesh (accessed Mar. 31, 2021).

[17] P. S. Kamdi and M. S. Deogade, "The hidden positive effects of covid-19 pandemic," International Journal of Research in Pharmaceutical Sciences, vol. 11, no. Special1, Mar. 2020, doi: 10.26452/ijrps.v11iSPL1.2712.

[18] "Exploring the impacts of COVID-19 on travel behavior and mode preferences," Transportation Research Interdisciplinary Perspectives, vol. 8, p. 100255, Nov. 2020, Accessed: Apr. 01, 2021. [Online].

[19] "Transportation in the Mediterranean during the COVID-19 pandemic era," Global Transitions, vol. 3, pp. 55-71, Jan. 2021, Accessed: Apr. 02, 2021. [Online].

[20] "Changes in local travel behaviour before and during the COVID-19 pandemic in Hong Kong," Cities, vol. 112, p. 103139, May 2021, Accessed: Apr. 01, 2021. [Online].

[21] "COVID-19 lockdown measures and travel behavior: The case of Thessaloniki, Greece," Transportation Research Interdisciplinary Perspectives, vol. 10, p. 100345, Jun. 2021, Accessed: Apr. 01, 2021. [Online].

[22] M. Gupta, N. M. Pawar, and N. R. Velaga, "Impact of lockdown and change in mobility patterns on road fatalities 
during COVID-19 pandemic," Transp. lett., pp. 1-14, Mar. 2021.

[23] S. R. Barnes, L.-P. Beland, J. Huh, and D. Kim, "The Effect of COVID-19 Lockdown on Mobility and Traffic Accidents: Evidence from Louisiana," GLO Discussion Paper, 616, 2020. Accessed: Apr. 01, 2021. [Online]. Available: http://hdl.handle.net/10419/222470.

[24] B. Sedain and P. R. Pant, "Road traffic injuries in Nepal during COVID-19 lockdown," F1000Res., vol. 9, no. 1209, p. 1209, Feb. 2021, Accessed: Apr. 01, 2021. [Online].

[25] U. Oguzoglu, "Covid-19 Lockdowns and Decline in Traffic Related Deaths and Injuries," May 2020, Accessed: Apr. 01, 2021. [Online]. Available: https://papers.ssrn.com/abstract=3608527.

[26] L. Lin, F. Shi, and W. Li, “Assessing Road Traffic Safety During COVID-19: Inequality, Irregularity, and Severity," Oct. 22, 2020.

[27] "Website.” https://doi.org/10.1016/j.aap.2020.105747 (accessed Mar. 24, 2021).

[28] "Impact of COVID-19 lockdown policy on homicide, suicide, and motor vehicle deaths in Peru," Prev. Med. , vol. 143, p. 106331, Feb. 2021, Accessed: Apr. 02, 2021. [Online].

[29] H. Inada, L. Ashraf, and S. Campbell, "COVID-19 lockdown and fatal motor vehicle collisions due to speed-related traffic violations in Japan: a time-series study," Inj. Prev., vol. 27, no. 1, pp. 98-100, Feb. 2021, Accessed: Mar. 31, 2021. [Online].

[30] "Bangladesh." https://www.ceicdata.com/en/bangladesh/motor-vehicle-registered/motor-vehicle-registeredbangladeshtotal\#: :text=Bangladesh's\%20Motor\%20Vehicle\%20Registered\%3A\%20Bangladesh,of\%20497\%2C374.000\%20Un it\%20for\%202018. (accessed Mar. 25, 2021).

[31] "Novel Coronavirus (COVID-19) Cases Data." https://data.humdata.org/dataset/novel-coronavirus-2019-ncov-cases (accessed Mar. 25, 2021).

[32] A. M. Almarashi and K. Khan, "Bayesian Structural Time Series," Nanosci. Nanotechnol. Lett., vol. 12, no. 1, pp. 5461, Jan. 2020.

[33] "CausalImpact." http://google.github.io/CausalImpact/ (accessed Mar. 25, 2021).

[34] Inferring the effect of an event using Causal Impact by Kay Brodersen. 2016.

[35] J. Cohen, Statistical Power Analysis for the Behavioral Sciences, 2nd Edition. Routledge, 1988.

[36] K. Nishida, "An Introduction to Causal Impact Analysis - learn data science," learn data science, Jun. $05,2017$. https://blog.exploratory.io/an-introduction-to-causal-impact-analysis-a57bce54078e (accessed Mar. 26, 2021).

[37] BBC News, "Covid-19: Record traffic around Paris as second French lockdown begins," BBC News, Oct. $30,2020$. https://www.bbc.com/news/world-europe-54742795 (accessed Mar. 31, 2021).

[38] J. Prasetijo et al., "Crash model based on integrated design consistency with low traffic volumes (due to health disaster (COVID-19)/movement control order)," Innovative Infrastructure Solutions, vol. 6, no. 1, pp. 1-7, Oct. 2020, Accessed: Apr. 01, 2021. [Online].

[39] "A descriptive analysis of the effect of the COVID-19 pandemic on driving behavior and road safety," Transportation Research Interdisciplinary Perspectives, vol. 7, p. 100186, Sep. 2020, Accessed: Mar. 31, 2021. [Online].

[40] "For many in Bangladesh, staying home isn't an option." https://www.unicef.org/coronavirus/many-bangladeshstaying-home-isnt-option (accessed Mar. 25, 2021).

[41] "Quantifying the impact of COVID-19 on non-motorized transportation: A Bayesian structural time series model," Transp. Policy, vol. 103, pp. 11-20, Mar. 2021, Accessed: Apr. 01, 2021. [Online].

[42] N. B. Mohammad, A. S. Huq. "Effects of COVID-19 Pandemic on Roadway Accident in Bangladesh", International Conference on Science and Technology for Celebrating the Birth Centenary of Bangabandhu (ICSTB-2021), Dhaka, Bangladesh, March 11-13, 2021. 